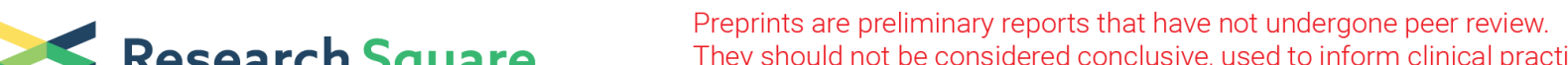 $\begin{array}{ll}\text { Research Square } & \text { They should not be considered conclusive, used to inform clinical practice, } \\ \text { or referenced by the media as validated information. }\end{array}$
}

\section{Differential Analysis of Expressed Genes in Diabetic Nephropathy Based on Bioinformatics Technology}

\section{Yu Liu}

Hospital of Chengdu University of Traditional Chinese Medicine

\section{Jundong Wang}

Hospital of Chengdu University of Traditional Chinese Medicine

wencheng Chi

First Affiliated Hospital of Heilongjiang University of Chinese Medicine

\section{Jing Xie}

Hospital of Chengdu University of Traditional Chinese Medicine

\section{Laikuan Teh}

Universiti Tunku Abdul Rahman

\section{Ling Wu}

Hospital of Chengdu University of Traditional Chinese Medicine

\section{Lihua Wu}

Hospital of Chengdu University of Traditional Chinese Medicine

\section{Luhan Qiao}

Chengdu Qingbaijiang District Traditional Chinese Medicine Hospital

\section{Meichao Yan}

Chengdu Qingbaijiang District Traditional Chinese Medicine Hospital

\section{Junye Deng}

Hospital of Chengdu University of Traditional Chinese Medicine

Mingquan Li ( $\sim$ L1563540954@163.com )

Hospital of Chengdu University of Traditional Chinese Medicine

\section{Research Article}

Keywords: diabetic nephropathy, bioinformatics, differentially expressed genes

Posted Date: August 5th, 2021

DOI: https://doi.org/10.21203/rs.3.rs-770285/v1

License: (c) (i) This work is licensed under a Creative Commons Attribution 4.0 International License. Read Full License 


\section{Abstract}

Objective: Bioinformatics technology was used in this study to analyze the expression data of patients with diabetic nephropathy (DN) and normal subjects from the microarray. The purpose of this study was to screen the differentially expressed genes in DN and to explore the pathogenesis and potential therapeutic targets of DN.

Methods: The data of gene expression in the gse142153 gene chip was downloaded from the gene expression database (GEO). The up-regulated and down-regulated expressed genes were analyzed by $\mathrm{R}$ language. The core genes of differentially expressed genes were analyzed by string database, Cytoscape software and its plug-in. The differentially expressed genes were analyzed by gene ontology and Kyoto Encyclopedia of genes and genomes.

Results: A total of 112 differentially expressed genes were screened, including 50 down-regulated genes and 62 up-regulated genes. There are 10 up-regulated core genes including CXCL8, MMP9, IL1B, IL6, IL10, CXCL2, CCL20, ATF3, CXCL3, F3. Their biological effects are mainly concentrated in the IL-17 signaling pathway, rheumatoid arthritis, viral protein interaction with cytokine and cytokine receptor, Amoebiasis, TNF signaling pathway, Legionellosis, Cytokine-cytokine receptor interaction, Lipid, and atherosclerosis, Malaria, NOD-like receptor signaling pathway, etc.

Conclusion: Analysis of differentially expressed genes and core genes enhanced the understanding of the pathogenesis of DN and provided a potential train of thought for the treatment of DN.

\section{Introduction}

Diabetic nephropathy is a major microvascular complication of diabetes. It is characterized by a continuous declination of renal function and the presence of albuminuria. This is commonly seen among patients with type 1 diabetes and types 2 diabetes with poor long-term glycemic control. Currently, the treatment for $\mathrm{DN}$ is mainly through regulating blood sugar, blood fat, blood pressure; reducing cardiovascular risk, and inhibiting the renin-angiotensin system (RAS). There is no effective way to prevent the progression of diabetic nephropathy. Therefore, clarifying the specific molecular mechanisms and therapeutic targets of diabetic nephropathy has become the problem we need to face[1].

In this study, the original chip data from the GEO database was extracted. The differentially expressed genes associated with diabetic nephropathy were excavated through bioinformatics methods to further explore the core genes, and to enrich the analysis of the function and pathways of the core genes. This will allow exploring the pathological mechanism and therapeutic targets of end-stage renal disease to provide ideas for the molecular mechanism and treatment of diabetic nephropathy.

\section{Method}

\subsection{Data extraction}


The expression data of gene fragment no. gse142135 was downloaded from gene expression database(http://www.ncbi.nlm.nih.gov/geo/). The data set includes 75 patients with end-stage renal disease and 40 normal subjects. The fragment platform was gpl6480agilent-014850 whole human genome microarray $4 x 44 k$ g4112f.

\subsection{Differential gene screening}

$\mathrm{R}$ language software was used to analyze between the normal group and diabetic nephropathy group to

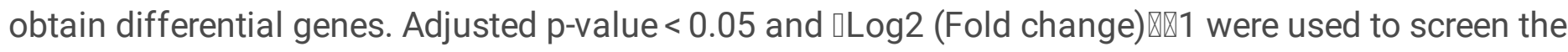
differentially expressed genes. The selected differentially expressed genes were analyzed using hierarchical cluster analysis. The volcano map and differential gene heat map were mapped to intuitively understand the differences in gene expression profiles.

\subsection{Core gene and functional annotation}

The differential genes are then analyzed through the string database at https://string-db.org/; proteinprotein interaction (PPI) analysis with screening criteria of combined score $>0.9$ and network analysis (cytohubba) to select the proteins with a high degree tend to be key proteins). These were carried out by three methods: cytohubba plug-in and mcode plug-in of Cytoscape software. Hence, the core genes were comprehensively screened by three network analysis methods with the selection criteria as: degree cutoff: 2 , node score cutoff: 0.2 , haircut: true, fluff: false, k-core: 2 , max. depth from seed: 100 . Then, the core genes were annotated, including gene function enrichment analysis (go gene ontology) and gene pathway enrichment analysis (KEGG, Kyoto Encyclopedia of genes and genomes). The R language "clusterprofiler" package and "ggplot2" package were used for go and KEGG enrichment analysis and visualization.

\section{Result}

\subsection{Based on the original gene fragment data of GSE142153,}

the differential gene with the absolute value of log2 (fold change) $>1$ and adjusted $P<0.05$ as the screening criteria, a total of 112 differentially expressed genes were obtained, including 50 downregulated genes and 62 up-regulated genes. Figure 1 shows the fire mountain map of differentially expressed genes and Fig. 2 show the heat map of differential gene expression

\subsection{After the first screening,}

a total of 50 down-regulated genes and 62 up-regulated genes were screened for core genes and functional annotation respectively. All these genes were analyzed for the PPI analysis using String Database and the combined score $>0.4$ was selected as the threshold of the PPI relationship. The relevant TSV format files were selected to obtain PPI relationship pair files. Then, the cytohubba plug-in 
of Cytoscape software was used to find the key targets and sub-networks of complex networks. Then, the module was analyzed by using the mcode plug-in of Cytoscape software. After cytohubba analysis, the top 10 key genes were defined according to the degree value, including up-regulated genes: CXCL8, MMP9, IL1B, IL6, IL10, CXCL2, CCL20, ATF3, CXCL3, F3; After mcode analysis, an important module was obtained: F3, IL1B, THBD, AREG, CCL20, MMP9, CXCL2, CXCL3, CXCL8 and IL10. Down regulated genes: TLR10, TLR7, CCR2, AIM2, GAPT, TNFAIP8L2, LRRN3, NRCAM, APAF1, HSPA1A; Mcode modules: TLR7, TLR10, AIM2, CCR2.

\subsection{After analyzing the differentially expressed genes,}

the up-regulated genes were found closely related to diabetic nephropathy. Three of the ten key genes were shown in Fig. 3. GO and KEGG analysis revealed that the up-regulated core genes were mainly related to cytokine activity $\llbracket c y$ tokine receptor binding $\square$ receptor ligand activity $\llbracket$ signaling receptor activator activity $\llbracket$ chemokine activity. It was enriched in producing IL-17 signaling pathway $[R$ Reumatoid arthritis $\square$ Viral protein interaction with cytokine and cytokine receptor $₫$ Amoebiasis $\square T N F$ signaling pathway $\square$ Legionellosis $\square$ Cytokine-cytokine receptor interaction $\square$ Lipid and atherosclerosis $\square$ Malaria $\square$ NOD-like receptor signaling pathway,etc.(Figs. 4 \& 5).

\section{Discussion}

Based on the latest statistics in the year 2017, a total of 451 million people have diabetes, and it is expected that by 2045, this figure will reach 693 million [2]. Diabetic nephropathy is one of the most serious microvascular complications. DN has become the main cause of the end-stage renal disease (ESRD) in many countries. The current treatment is not managed to cure DN and prevent the progression to ESRD. In recent years, bioinformatics data have been widely used to study the mechanism of disease and to predict the possible therapeutic targets. In this study, bioinformatics was used to analyze the gene expression between patients with diabetic nephropathy and normal subjects. Through screening of core genes and gene annotation, the up-regulated differentially expressed genes were significantly enriched in immune inflammatory-related signaling pathways. Thus, this implicated that the function of differentially expressed genes is closely related to the signal pathway of immune inflammation. This attributed that the pathological mechanism of diabetic nephropathy is mainly caused by inflammatory stimulation leading to cell damage. The risk factors for the release of renal cells can trigger the remodeling process by stimulating the renal cells and activating the immune cells of the congenital and adaptive system[3].

In this study, GO and KEGG were used to annotate the core genes. Consistent finding was found in which the core genes were mainly related to the immune-inflammatory signaling pathway. This had further suggested that the immune system involving in the inflammatory mediating process reaction is the key factor in the pathogenesis of diabetic nephropathy[4]. The key genes concerning DN were CXCL-8, MMP9, IL-1B, IL-6, and ATF3. 
CXCL8 (IL-8) is a cytokine derived from a variety of cells and has a variety of biological functions. It can induce leukocyte chemotaxis and activation, lysosomal release activation and chemotaxis. High glucose can stimulate the production of CXCL8 and excrete into the urine, which is significantly correlated with the level of $\mathrm{HbA} 1 \mathrm{c}$, but not with the stage of disease or pathological damage. Based on the finding, we may stipulate that CXCL8 will be increased in the early stage of diabetic nephropathy to evaluate the degree of renal damage in patients with type 2 diabetic nephropathy[5-6].

MMP-9, the ninth member of the MMP family, may play an important role in leukocyte migration and local proteolysis of the extracellular matrix. Many studies have shown that MMP-9 is an important inflammatory marker involved in the pathophysiological process of DN. Studies have shown that MMP9 can delay glomerulosclerosis and interstitial fibrosis by increasing ECM degradation, thereby slowing down the progression of DN [7].

IL-1B is one of the three cytokines produced by interleukin-1, which is involved in a variety of inflammatory processes. It is related to IgA nephropathy, diabetic nephropathy and other kidney diseases. Studies have shown that the relationship between IL-1B and DN can be explained by the biological characteristics of IL-1. IL-1 is an important proinflammatory cytokine and is increased in diabetic nephropathy. Monika Buraczynska suggested that in diabetic nephropathy patients and mouse models, the activation of IL-1 involved in the regulation of cytokine production and the activation of extracellular matrix may play an important role in the progression of renal failure in diabetic patients [8].

IL-6 is a cytokine with multiple functions, which plays an important role in inflammation and immune response. Senthilkumar et al. found that IL-6 levels in diabetic nephropathy patients were significantly higher [9].

ATF3 is a stress-induced transcription factor, which is transiently expressed after stimulation. Studies have shown that in diabetic nephropathy, the expression of AFT3 is significantly increased [10].

\section{Conclusions}

This study suggested up-regulated genes were involved in the differential expression of DN. The upregulated genes were mainly involved in many biological processes of the inflammatory immune response, involving the IL-17 signaling pathway, rheumatoid arthritis, viral protein interaction with cytokine, and signaling pathway. Among them, the core gene may be one of the causes of diabetic nephropathy. It provides potential information to understand the target gene for the follow-up study of the molecular mechanism and drug research of diabetic nephropathy.

\section{Declarations}

\section{Availability of data and materials}


The dataset(s) supporting the conclusions of this article are available in GEO database (https://www.ncbi.nlm.nih.gov/geo/).

\section{Author contributions}

Thanks to Yu Liu for writing this article『Professor Mingquan Li囚Jundong Wang for providing the article ideas, Wencheng ChiðJing Xie for their help in the article modification.Laikuan teh's help in language polishing囚Ling Wu and Lihua Wu are looking for help from the literature.Luhan Qiao®Meichao Yan and Junye Deng help collect data.

\section{Conflicts of interest}

None

\section{Acknowledgement}

Not applicable

\section{Declaration}

None

\section{Funding/support}

None

\section{Ethics approval}

None

\section{References}

1. Hudspeth, J. Diabetic Kidney Disease. Prim Care, 47 (4), 645-659 (2020 Dec;). Upadhyay A

2. Cho, N. H. et al. IDF Diabetes Atlas: Global estimates of diabetes prevalence for 2017 and projections for 2045.Diabetes Res. Clin. Pract.2018;138:271-281.

3. Zheng, Z. Immune Cells and Inflammation in Diabetic Nephropathy. J Diabetes Res, 2016, 1841690 (2016). Zheng $F$ 
4. Petrica, L. Pro-inflammatory cytokines are associated with podocyte damage and proximal tubular dysfunction in the early stage of diabetic kidney disease in type 2 diabetes mellitus patients. $J$ Diabetes Complications, 34 (2), 107479 (2020 Feb;). Vlad D

5. Fiorina, P. The IL-8-CXCR1/2 axis contributes to diabetic kidney disease. Metabolism, 4, 154804 (2021 Jun). Niewczas MAEl Essawy B

6. Tomino, Y. Urinary levels of monocyte chemoattractant protein-1 (MCP-1) and interleukin-8 (IL-8), and renal injuries in patients with type 2 diabetic nephropathy. J Clin Lab Anal, 16 (1), 1-4 (2002). Shirato I

7. Matrix Metalloproteinase-9 - 1562C/T Gene Polymorphism Is Associated with Diabetic Nephropathy \& Chang, L. Association of MMP-9 polymorphisms with diabetic nephropathy risk: A protocol for systematic review and meta-analysis. Med. (Baltim), 18;99((38), e22278 (2020 Sep). Chen G

8. Wacinski, P. Interleukin-1 $\beta$ Gene (IL1B) Polymorphism and Risk of Developing Diabetic Nephropathy. Immunol Invest, 48 (6), 577-584 (2019 Aug;). Zaluska W

9. Packirisamy, R. M. Role of omentin 1 and IL-6 in type 2 diabetes mellitus patients with diabetic nephropathy. Diabetes Metab Syndr, 12 (1), 23-26 (2018 Jan-Mar;). Bobby Z

10. Zhang, B. Inducible ATF3-NFAT axis aggravates podocyte injury. J Mol Med (Berl), 96 (1), 53-64 (2018 Jan;). Shi W

\section{Figures}




\section{Volcano}

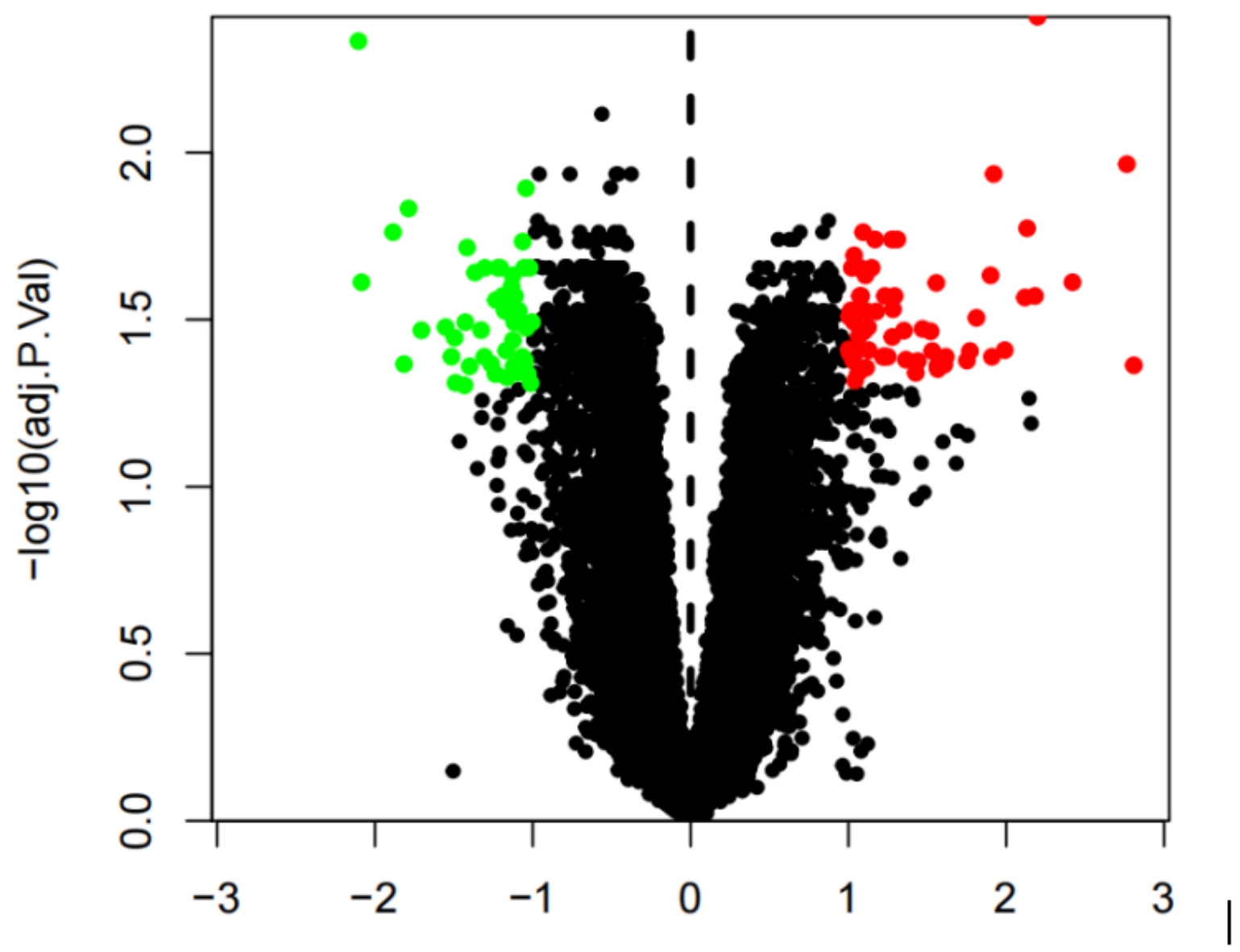

Figure 1

Volcano map of differentially expressed genes 


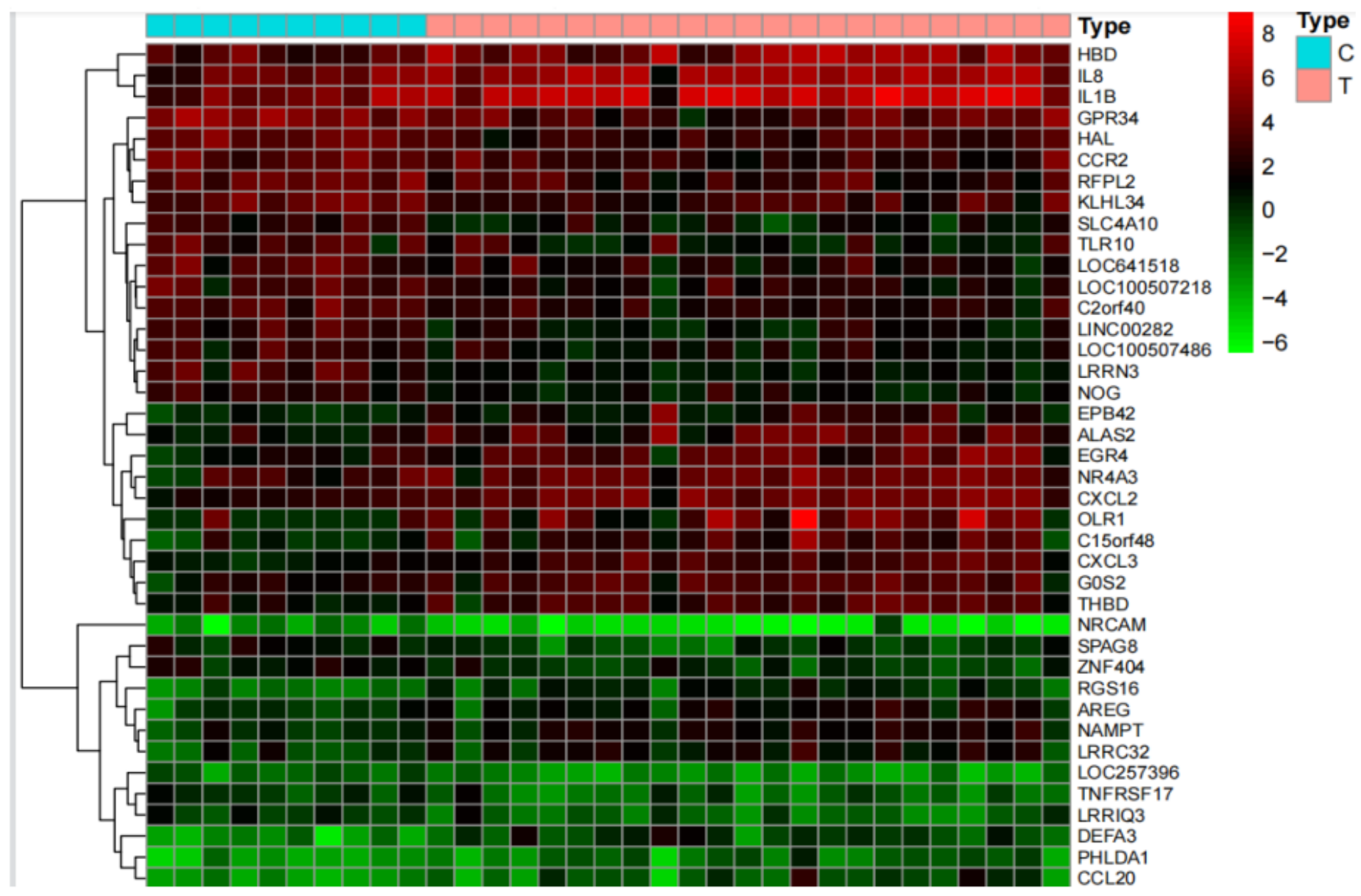

Figure 2

heat map of differential gene expression 


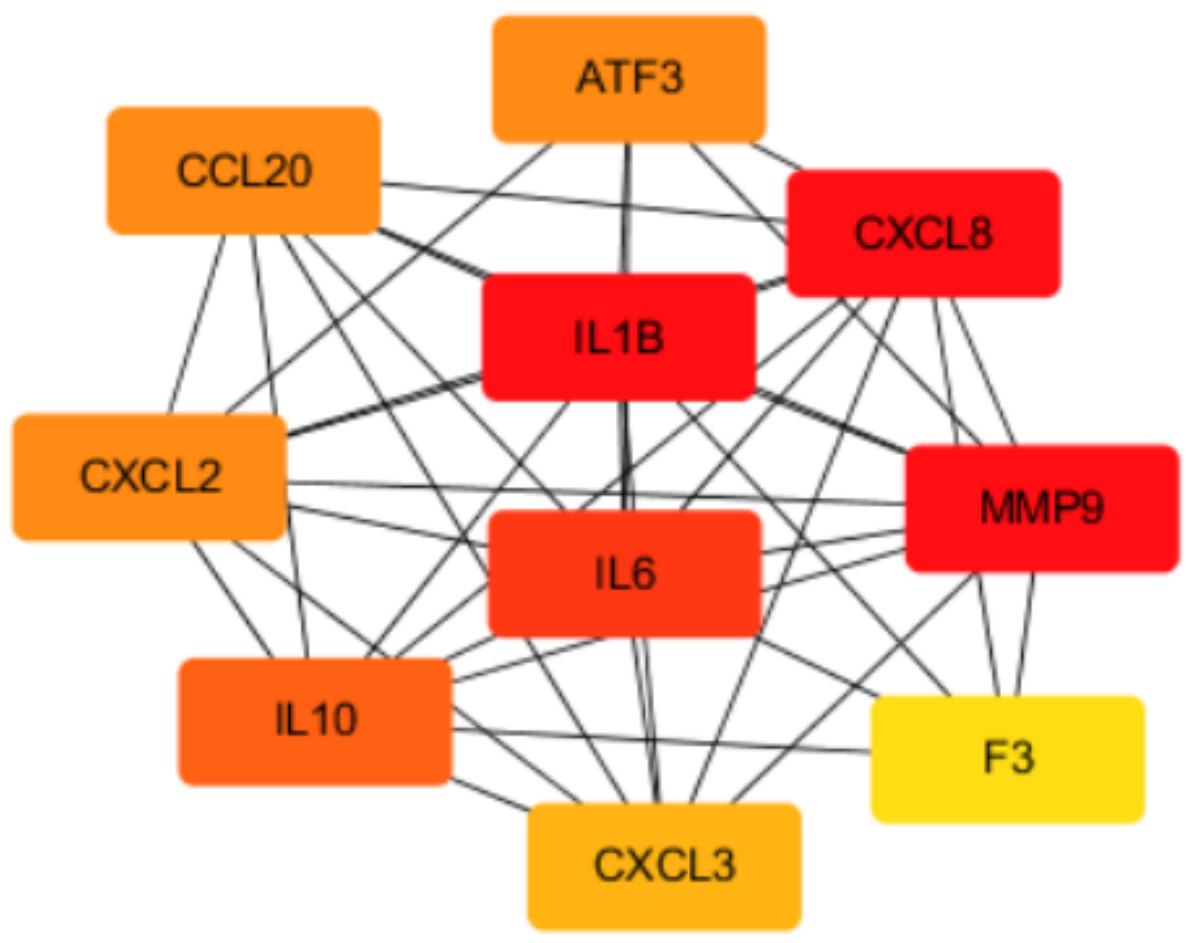

Figure 3

Key gene network 


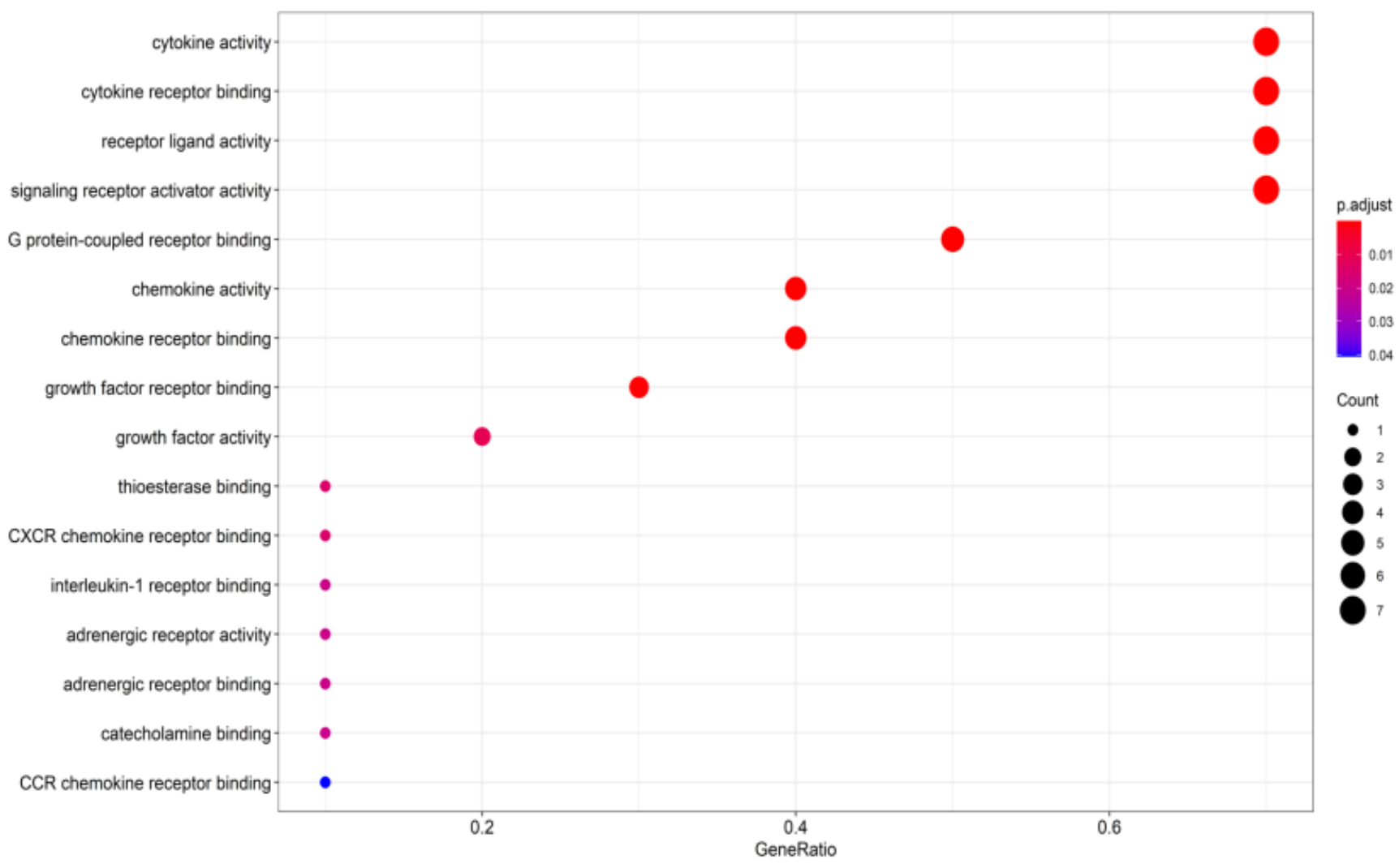

Figure 4

enrichment analysis of key gene GO 


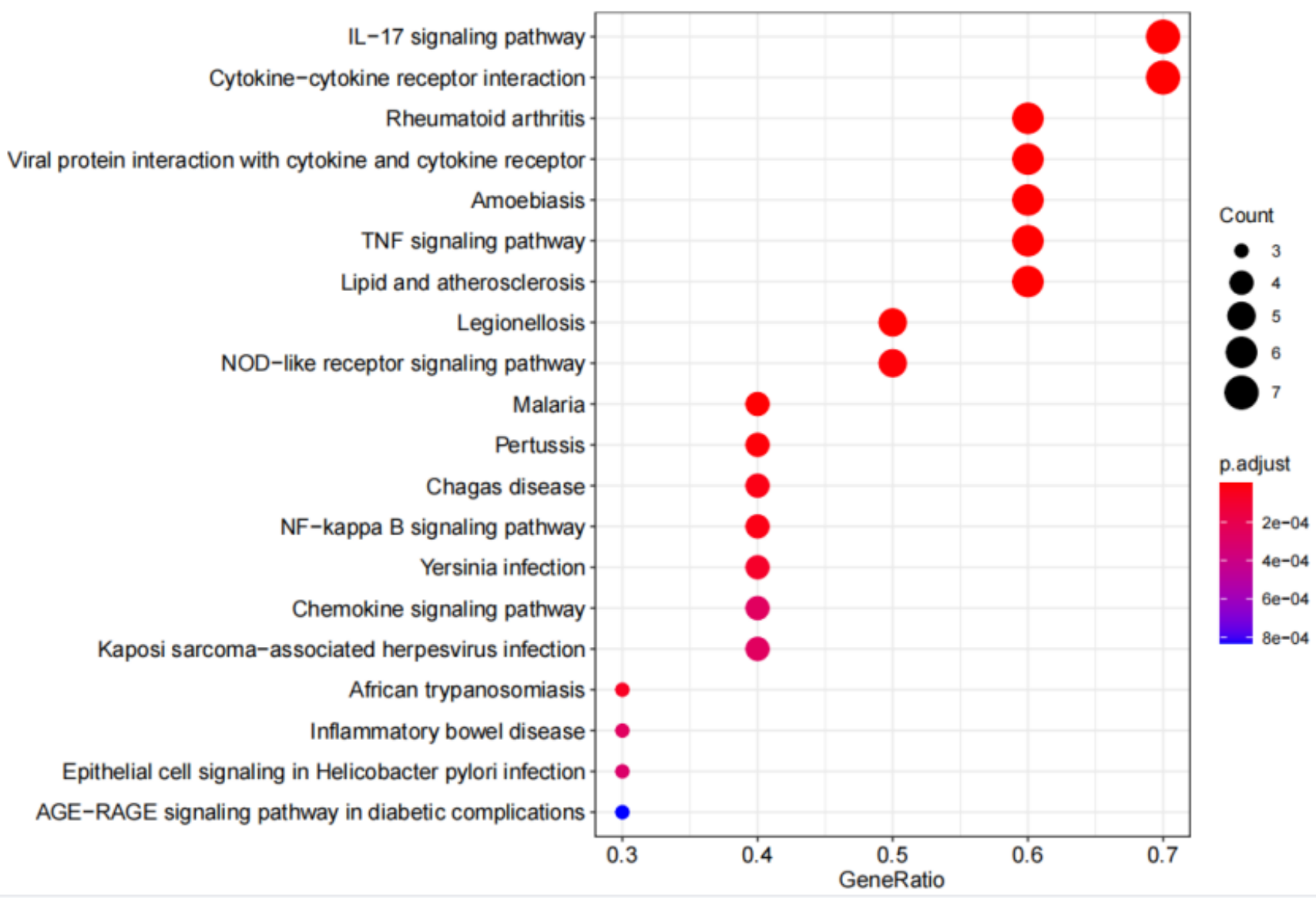

Figure 5

enrichment analysis of key gene KEGG 